\title{
ON THE FINITE DIMENSIONALITY OF EVERY IRREDUCIBLE UNITARY REPRESENTATION OF A COMPACT GROUP
}

\section{LEOPOLDO NACHBIN}

We shall prove that every irreducible unitary representation of a compact group is finite dimensional. Our argument is a variation of known proofs and it hardly could be based on an idea different from those already current. It makes no use of the Peter-Weyl theorem and of compact or Hilbert-Schmidt operators and seems simpler than the proofs in $[1 ; 2 ; 3 ; 4]$. Its crucial point is that the prospectively finite dimension of the representation Hilbert space is expressible by a known integral formula.

Let $\mathcal{F} \neq 0$ be a Hilbert space and $x \rightarrow U_{x}$ be a group homomorphism of a compact group $G$ into the group $U(\mathcal{H C})$ of all unitary operators in $\mathfrak{F}$, such that the scalar product $\left(\xi \mid U_{x} \eta\right)$ is a continuous function of $x \in G$ for all $\xi, \eta \in \mathcal{H}$. Suppose that this representation is irreducible, namely that there is no closed vector subspace of $\mathcal{H}$ invariant under all $U_{x}$ except the trivial ones 0 and $\mathfrak{H}$. Then there results that $\mathfrak{H C}$ is finite dimensional. In fact, let $\xi, \eta, \xi^{\prime}, \eta^{\prime} \in \mathcal{H}$. Denoting complex conjugation by a star, since

$$
\left|\int\left(\xi \mid U_{x} \eta\right) \cdot\left(\xi^{\prime} \mid U_{x} \eta^{\prime}\right)^{*} d x\right| \leqq\|\xi\| \cdot\|\eta\| \cdot\left\|\xi^{\prime}\right\| \cdot\left\|\eta^{\prime}\right\|,
$$

there is an operator $T$ on $\mathcal{F}$ depending on $\eta, \eta^{\prime}$ such that

$$
\int\left(\xi \mid U_{x} \eta\right) \cdot\left(\xi^{\prime} \mid U_{x} \eta^{\prime}\right)^{*} d x=\left(T \xi \mid \xi^{\prime}\right) .
$$

$T$ commutes with every $U_{t}$ since

$$
\begin{aligned}
\left(T U_{t} \xi \mid \xi^{\prime}\right) & =\int\left(\xi \mid U_{t-1} \eta\right) \cdot\left(\xi^{\prime} \mid U_{x} \eta^{\prime}\right)^{*} d x \\
& =\int\left(\xi \mid U_{x} \eta\right) \cdot\left(\xi^{\prime} \mid U_{t x} \eta^{\prime}\right)^{*} d x=\left(T \xi \mid U_{t}^{*} \xi^{\prime}\right)=\left(U_{t} T \xi \mid \xi^{\prime}\right),
\end{aligned}
$$

from which $T U_{t}=U_{t} T$ follows. The irreducibility of the representation then implies that $T$ is a scalar operator, that is $T=\lambda\left(\eta, \eta^{\prime}\right) I$ and we get

$$
\int\left(\xi \mid U_{x} \eta\right) \cdot\left(\xi^{\prime} \mid U_{x} \eta^{\prime}\right)^{*} d x=\lambda\left(\eta, \eta^{\prime}\right)\left(\xi \mid \xi^{\prime}\right) .
$$

Received by the editors February 29, 1960. 
By interchanging the roles of the couples $\left(\xi, \xi^{\prime}\right)$ and $\left(\eta, \eta^{\prime}\right)$ and using the rule $\int f\left(x^{-1}\right) d x=\int f(x) d x$, we get

$$
\lambda\left(\eta, \eta^{\prime}\right)\left(\xi \mid \xi^{\prime}\right)=\lambda\left(\xi, \xi^{\prime}\right)^{*}\left(\eta \mid \eta^{\prime}\right)^{*} .
$$

Hence $\lambda\left(\eta, \eta^{\prime}\right)=c\left(\eta \mid \eta^{\prime}\right)^{*}$, where $c$ is a constant, and

$$
\int\left(\xi, U_{x} \eta\right) \cdot\left(\xi^{\prime} \mid U_{x} \eta^{\prime}\right)^{*} d x=c\left(\xi \mid \xi^{\prime}\right) \cdot\left(\eta \mid \eta^{\prime}\right)^{*} .
$$

If we let $\xi, \eta, \xi^{\prime}, \eta^{\prime}$ all become equal to a unit vector $\alpha$, we get

$$
c=\int\left|\left(\alpha \mid U_{x} \alpha\right)\right|^{2} d x .
$$

Hence $c>0$, since the positive continuous function whose integral is $c$ has strictly positive value at the identity of $G$.

Now let $e_{1}, \cdots, e_{n}$ be orthonormal in 3 C. Let $\eta, \eta^{\prime}$ become equal to $e_{i}$ and $\xi, \xi^{\prime}$ become equal to $\alpha$ in (1). By adding the resulting equalities and using Bessel's inequality

$$
\sum_{i=1}^{n}\left(\alpha \mid U_{x} e_{i}\right) \cdot\left(\alpha \mid U_{x} e_{i}\right)^{*} \leqq\|\alpha\|^{2}=1
$$

since $U_{x} e_{1}, \cdots, U_{x} e_{n}$ are orthonormal, we get $n c \leqq 1$, that is $n \leqq 1 / c$. This completes the proof that the dimension of $\mathfrak{F C}$ is finite.

We remark that, if $n$ is supposed to be the finite dimension of $\mathscr{H}$, then (2) holds as an equality and so we get $n c=1$, that is $c=1 / n$. Then (1) becomes a known formula (see [5, Chapter V]) which we took as motivation for the above proof.

\section{REFERENCES}

1. F. Bruhat, Algèbres de Lie et groupes de Lie, Textos de Matemática no. 3, Recife (1959).

2. P. Cartier, Structure topologique des groupes de Lie généraux, Séminaire Sophus Lie, 1e année, Exposé 22, Paris (1955).

3. A. Hurevitsch, Unitary representations in Hilbert space of a compact topological group, Rec. Math. (Mat. Sbornik) n.s. vol. 13 (55) (1943) pp. 79-86.

4. P. Koosis, An irreducible unitary representation of a compact group is finite dimensional, Proc. Amer. Math. Soc. vol. 8 (1957) pp. 712-715. 1940.

5. A. Weil, L'intégration dans les groupes topologiques et ses applications, Paris,

Instituto de Matematica Pura e Aplicada

Centro Brasileiro de Pesquisas Fisicas

Rio de JaNeiro, BrazIL 